\title{
Research on wearable intelligent publicity system based on interactive technology
}

\author{
Zixin Huang $^{1, a}$, Weiping Luo ${ }^{1, b}$ and Zhao $\mathrm{Ai}^{2, \mathrm{c}}$ \\ ${ }^{1}$ School of Mechanical Engineering and Automation, Wuhan Textile \\ University, Wuhan, China, 430200 \\ ${ }^{2}$ School of Electronic and Electrical Engineering, Wuhan Textile \\ University, Wuhan, China, 430200 \\ a:E-mail:390090440@qq.com, b:E-mail: 651871236@qq.com, c:E-mail: \\ 250896931@qq.com
}

\begin{abstract}
In this paper by means of interactive concept and interactive technology to propagandize as a focus and searching for intelligent publicity system designing and business model. To design a new interactive media technology based on the research, user friendly and satisfy customers to achieve high conversion rate and high efficiency can be wearable intelligent publicity system. And discusses the design of hardware and software can wearable intelligent publicity system. The hardware system with ARM9 designed by microcontroller, GPS positioning system module; software mainly includes user workload assessment system and the interface of LabVIEW development. And GPRS, real-time interactive system using $\mathrm{PC}$ as a person wearing end processing unit and a micro controller.

Keywords: interactive technology; wearable intelligent publicity system; ARM9; GPS positioning; GPRS.

\section{Introduction}

With the increasing development of information technology, science and technology to infiltrate all aspects of life ${ }^{[1]}$. Close combination of clothing and technology will usher in a new era of garment industry in the future. Electronic intelligent garment has good appearance and serviceability, comfort and maintainability while meet the clothing, but also has a unique function. Will be high-tech information technology and garment front together, the physical world
\end{abstract}


is composed of sensors, actuators, display and computer elements. These elements are seamlessly integrated into our lives and provides intelligent environment the digital world interesting through uninterrupted network connection together and bringing a new more comfortable and convenient life the way for $\mathrm{us}^{[2]}$. The fashion electronic products as clothing and clothing accessories and make them to be the high degree of integration. And make embedded system hardware the more intelligent. There are also includes other supporting innovation of textile materials, graphic design, sensing and wireless transmission technology. In order to better complete the function of advertising. It will accompany at any time to transfer the latest information in our daily life, like to wear as much as possible into the surrounding environment ${ }^{[3]}$.

\section{Interaction Design}

1) Interaction between PC and the ARM9 core Dashboard. PC advertising content will be transmitted to the ARM9 core Dashboard and the user's data also be transmitted to the PC by network communication.

2) Interaction between the core Dashboard and hardware modules. The core Dashboard can transmit ads content to screen and mini voice box. At the same time, the GPS positioning transmit the user's data to it.

3) Interaction between the hardware and the carrier cloth. There are more advantage for real time transmission of interaction design that facilitate information and is convenient for hardware maintained.

\section{Hardware Design}

Fig.1 show us that the system hardware consists of ARM9 core control panel, display screen, mini voice box, GPS positioning, GPRS, power module, PC. The ARM9 hardware circuits plays a central role in the whole circuit. The PC sent the signal to the intelligent GPRS through the wireless network, then GPRS module sent the signal to the core Dashboard by wireless network. Then the core Dashboard control the screen to display the advertisement. At the same time, the 
core Dashboard control the mini voice box to work. When the user starts to work, start the GPS positioning system and transmit it's data to the core Dashboard. At last, after the controller processing. The data via GPRS transmit back to the PC.

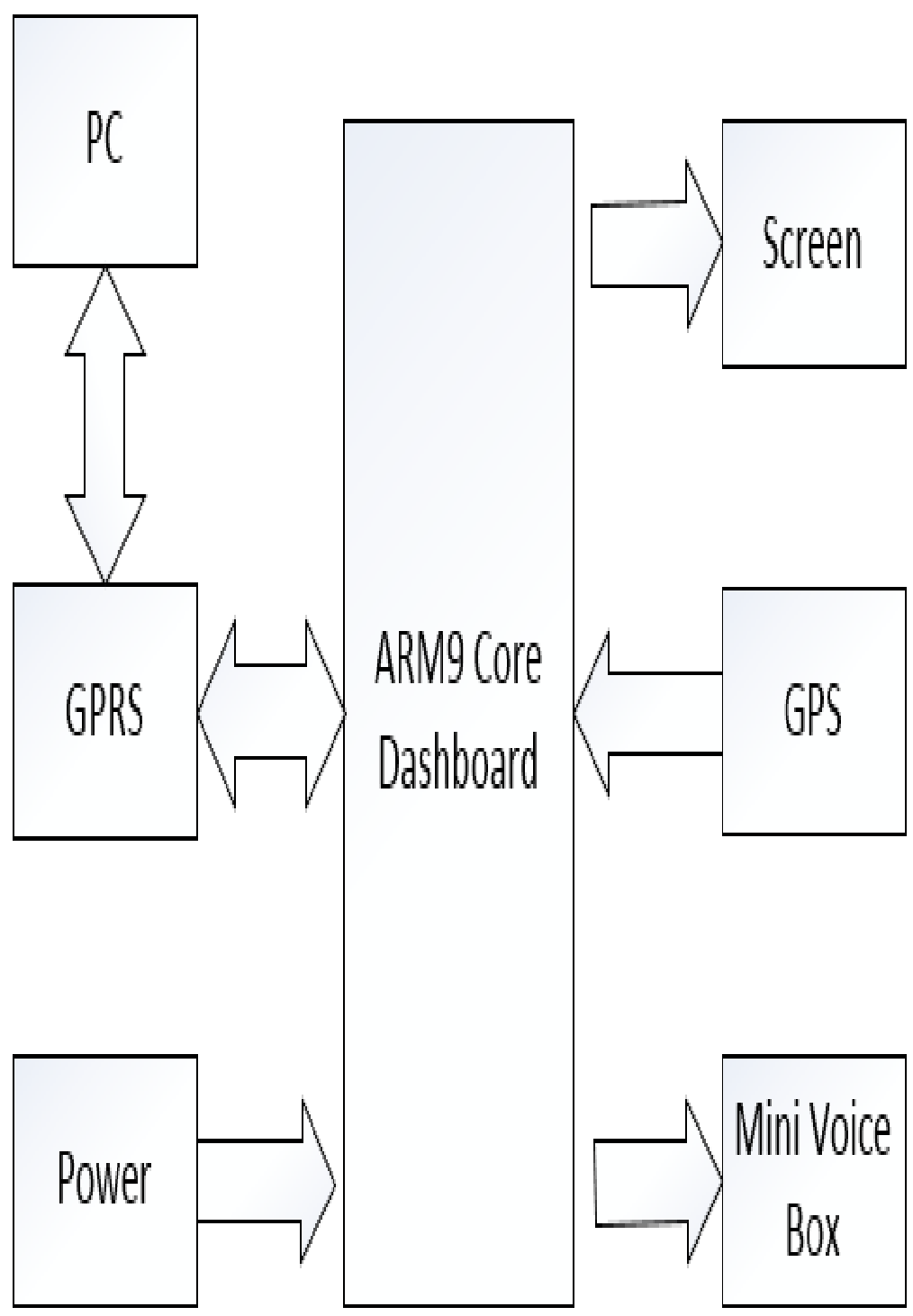

Fig.1 The circuit system design 


\section{Software Design}

The system's software was divided into two parts: PC, the ARM9 core Dashboard (Fig.2). The ARM9 core Dashboard obtained the GPS positioning of latitude and longitude by GPRS and then transmitted the acquired information to the PC's LabVIEW ${ }^{[4]}$ program through the network communication. And the LabVIEW program did the data comparison and made a determination. After that, the correct information is updated to the user workload database in real-time and the LabVIEW program sent the data through the network to user workload assessment system. According to the information from the user workload assessment system, the user can obtain the corresponding reward.

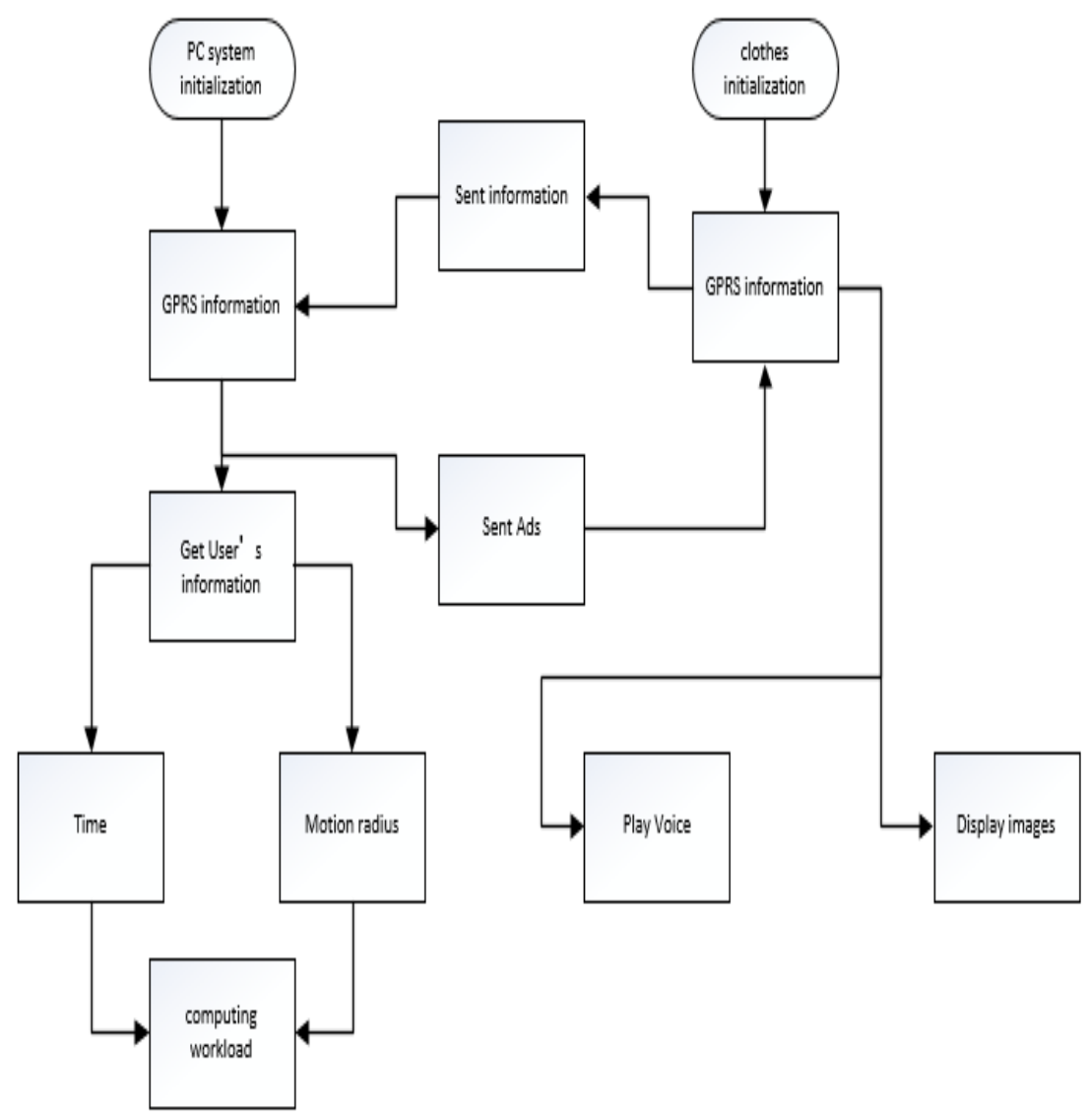

Fig.2 the Software flowchart 


\section{Experiment and Result}

At first, the PC will transmit advertising content to the ARM9 core Dashboard by GPRS. The core Dashboard will sent content to the display screen and mini sound to broadcast communication of advertisement information. At the same time, the timer starts open user work time T. GPS positioning system open to record the user's range of motion, to the user's starting point as origin establish Movement Function as shown in Fig.3, and real-time transmits the data back to the controller process, the processed data back to Working background via GPRS. The PC interface can show track user route. In the effective time of $\mathrm{T}$ activity and the effective radius of the $\mathrm{R}$ ruled the workload of the user, which can just give the user pay ${ }^{[5]}$. Each piece of wearable intelligent publicity system are a unique IP address, can become an Internet portal. When the user wear smart clothing activity in the crowd is advertisement, the ubiquitous Internet access. Make full use of the algorithm is of big data recognition and data mining based on cloud computing, analysis system, the intelligent wear real-time data processing equipment to the clouds, convert data into visual information, provide the basis for the analysis of the working background.

With the increasingly development of information technology, science and technology to infiltrate every aspect of life and application of science and technology brings human pleasure and good experience. At the same time, people, technology, application environment of interaction design will be more and more into people's horizons. Combined closely with the clothing and technology will create a new era of garment industry in the future.

The combination of interaction design and smart clothing is inevitable in the development of both, interaction design is bound to the development of more and more close relationship with people's life, and intelligence is dress of dress one of development direction. The wearable intelligent publicity system dress cannot lack of intelligent interactive technology support and integrated into the interactive design thinking. So, interaction design and the wearable intelligent publicity system clothing both are mutually reinforcing relationship, a combination of both is inevitable in the development of information society. 


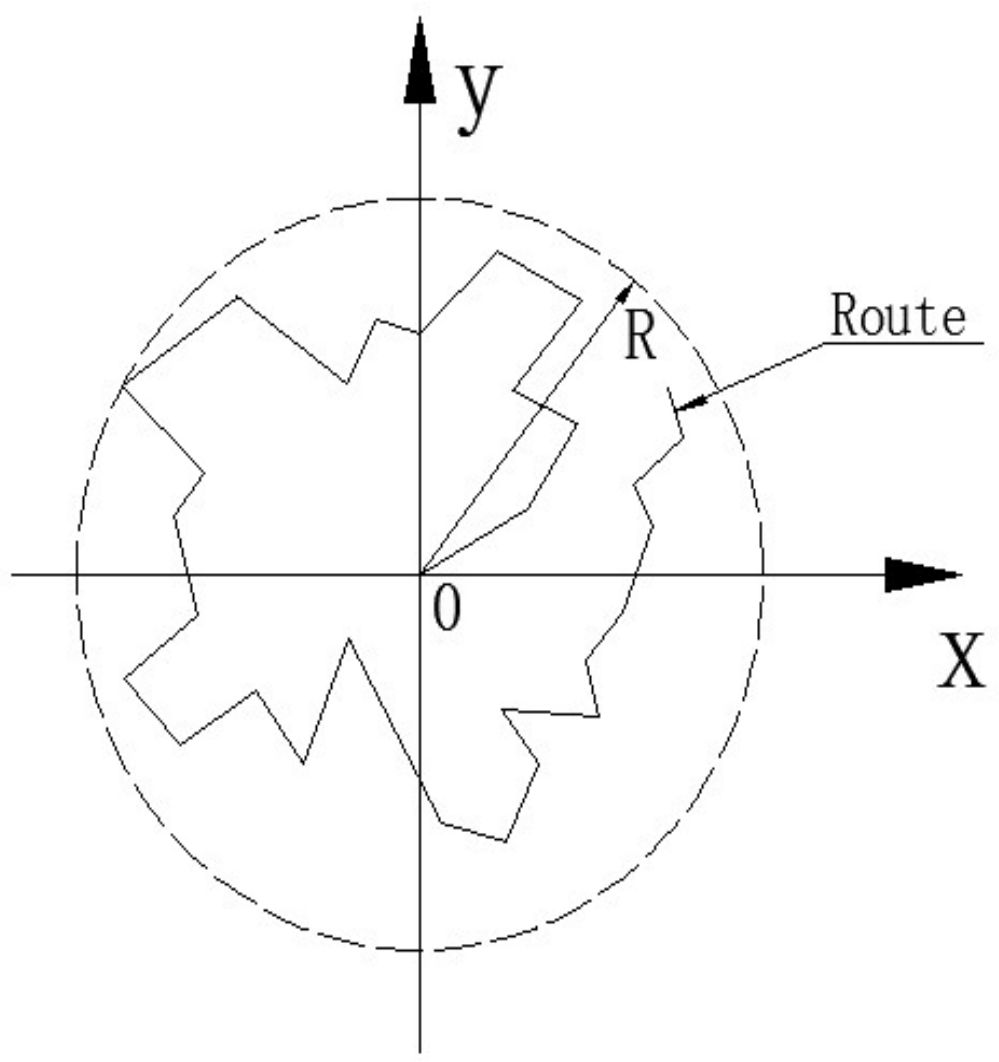

Fig.3 the Movement Function

\section{Conclusions}

In a word, using Internet service thinking to service users are not only smart wearable device the future development ideas, but also each manufacturer direction. Although smart wearable equipment popularization takes time, but with the portable, intelligent wearable device cloud interconnection performance advantage more and more obvious. The diversity of advertisement information and transfer accuracy demand of people is increasingly rising a perfect combination of the two will undoubtedly become the power intelligent wear equipment market explosive growth. For the wearable intelligent publicity system, sales of equipment is not enough, only more important by big data from all walks of life to explore new business model, and it involves smart ads apparel 
manufacturers and the customer's choice of development pattern, and the biggest problem of current smart apparel enterprises. Therefore, in the local market segment in the process of continuously, gradually explore the user, wearable intelligent publicity system operator and advertising customer tripartite win-win business model is the driving force for the development of the wearable intelligent publicity system.

\section{Acknowledgements}

1. Weiping Luo is corresponding author

2. Chinese Textile Industry Association of science and technology guidance project

(Grant No. 2010054)

3. The open project of Key Laboratory of digital textile equipment of Hubei Province, China (Grant No. DTL200909)

4. Xinchang county science and technology project of Hubei Province, China (Grant No. 201209)

\section{References}

[1] L.Meng., Y.Liu., 2011., Preschool children clothing design based on Intelligent Interaction Technology Research[J]. HUNDRED SCHOOLS IN ARTS, 5, 231-234.

[2]Y. H.Zhu., B. Y.Xu., 2013., Study on technology and application of intelligent wear[J]. SCIENCE AND TECHNOLOGY INFORMATION, 31, 26-27. [3]M. J.Jiang., 2014., Thoughts and suggestions about the prospect of intelligent wearable device development[J]. CHINA INTERNATIONAL INFORMATION AND COMMUNICATION DEVELOPMENT ISSUE, 17, 64-68.

[4]K. Cor J., 1995, LabVIEW: a software system for data acquisition, data analysis, and instrument control[J]. Knowing Your Monitoring Equipment. 11, 51-58

[5]Z. X.Huang, China. Patent 201420393609.5. (2013)

[6] Seyam Abdelfattah M,Ph D. Electrifying opportunities[J].Textile 
Word,2003,2: $30-32$

[7]Adrian Wilson. Pioneers of smart fabrics have grounds for optimism[J].Textile and Apparel, 2008,10:44-46.

[8]Noel Palomo-Lovinski. The future of digital clothing[J]. Clothing and Textiles Research Journal, 2008,26:121-125.

[9]Tao XM. Wearable electronic and photonics[M].England: Woodhead Publishing Limited, 2004:198-214. 